\title{
REVIEW
}

\section{Potential synergy between tau aggregation inhibitors and tau chaperone modulators}

\author{
Laura J Blair, Bo Zhang and Chad A Dickey*
}

\begin{abstract}
Tau is a soluble, microtubule-associated protein known to aberrantly form amyloid-positive aggregates. This pathology is characteristic for more than 15 neuropathies, the most common of which is Alzheimer's disease. Finding therapeutics to reverse or remove this non-native tau state is of great interest; however, at this time only one drug is entering phase III clinical trials for treating tauopathies. Generally, tau manipulation by therapeutics can either directly or indirectly alter tau aggregation and stability. Drugs that bind and change the conformation of tau itself are largely classified as aggregation inhibitors, while drugs that alter the activity of a tau-effector protein fall into several categories, such as kinase inhibitors, microtubule stabilizers, or chaperone modulators. Chaperone inhibitors that have proven effective in tau models include heat shock protein 90 inhibitors, heat shock protein 70 inhibitors and activators, as well as inducers of heat shock proteins. While many of these compounds can alter tau levels and/or aggregation states, it is possible that combining these approaches may produce the most optimal outcome. However, because many of these compounds have multiple off-target effects or poor blood-brain barrier permeability, the development of this synergistic therapeutic strategy presents significant challenges. This review will summarize many of the drugs that have been identified to alter tau biology, with special focus on therapeutics that prevent tau aggregation and regulate chaperone-mediated clearance of tau.
\end{abstract}

\section{Review}

\section{Therapeutic targeting of tau triage}

Tauopathies, a class of neurodegenerative diseases including Alzheimer's disease, frontotemporal dementia, and progressive supranuclear palsy, are characterized by the pathological aggregation of hyperphosphorylated tau tangles in the human brain [1]. Because aberrant protein accumulation is a hallmark of many neurological diseases, and tau is one of many proteins that form diseaseassociated aggregates, this can present a new challenge for finding an aggregation inhibitor specific for tau.

Studies have shown that several molecular chaperone families, known as heat shock proteins (Hsps), are involved with preventing tau aggregation $[2,3]$ or assisting in tau degradation [4]. These families, named for their general protein size in kiloDaltons, include Hsp70 and

* Correspondence: cdickey@health.usf.edu

Department of Molecular Medicine, University of South Florida, 4001

E. Fletcher Avenue, MDC 36, Tampa, FL 33613, USA
Hsp90, the smaller Hsp40, and small Hsps. Recently, a number of small molecule inhibitors have been developed and studied for their roles in regulating the ATPase activities of Hsp70 and Hsp90. In addition, much of the drug discovery efforts directed at tau are aimed at disrupting its aggregation; several aggregation inhibitors have been identified and their potential efficacy has been shown using model systems. This review will discuss drugs that have been developed to modulate the chaperone repertoire, as well as recent advances in therapeutics affecting tau aggregation. Table 1 summarizes all of the drugs discussed in this review. We speculate that these compounds could be synergistic, such that aggregation disruption followed by tau clearance could be more beneficial than either effect alone. By creating more soluble tau through inhibiting its aggregation, chaperones have a greater opportunity to bind to tau. This chaperone-bound tau can then be targeted for degradation. 
Table 1 List of drugs

\begin{tabular}{|c|c|c|c|c|c|}
\hline Tau aggregation inhibitors & Family & Mechanism & Toxicity & BBB permeability & Other notes \\
\hline AQ2S & Anthraquinone & $\beta$-sheet inhibitor & Nontoxic & Likely & Laxative \\
\hline Emodin & Anthraquinone & $\beta$-sheet inhibitor & Nontoxic & Likely & Laxative \\
\hline Daunorubicin & Anthraquinone & $\beta$-sheet inhibitor & Nontoxic & Likely & Laxative \\
\hline Mitoxantrone & Anthraquinone & $\beta$-sheet inhibitor & Nontoxic & Likely & Laxative \\
\hline Pixantrone & Anthraquinone & $\beta$-sheet inhibitor & Nontoxic & Likely & Laxative \\
\hline $\mathrm{C} 11$ & Carbocyanine & $\beta$-sheet inhibitor & Low toxicity & Likely & \\
\hline N744 & Carbocyanine & $\beta$-sheet inhibitor & Low toxicity & Unknown & \\
\hline PR-619 & Diaminopyridine & Hsp70 inhibitor & Some toxicity & Unknown & \\
\hline 17-AAG & Natural product derivative & Hsp90 inhibitor & Some toxicity & Permeable & Low bioavailability \\
\hline Geldanamycin & Natural product & Hsp90 inhibitor & Highly toxic & Not permeable & Toxic \\
\hline Hydroxytyrosol & Natural product & $\beta$-sheet inhibitor & Nontoxic & Permeable & \\
\hline Novobiocin & Natural product & Hsp90 inhibitor & Low toxicity & Poorly permeable & \\
\hline Oleuropein & Natural product & $\beta$-sheet inhibitor & Nontoxic & Not permeable & \\
\hline Oleuropein aglyxone & Natural product & $\beta$-sheet inhibitor & Nontoxic & Unknown & \\
\hline Radicicol & Natural product & Hsp90 inhibitor & Some toxicity & Poorly permeable & Low bioavailability \\
\hline Withaferin A & Natural product & Cdc37 inhibitor & Some toxicity & Permeable & \\
\hline Paclitaxel & Natural product & Microtubule stabilizer & Highly toxic & Poorly permeable & \\
\hline Curcumin & Natural product; flavonoid & Hsp70 inhibitor & Nontoxic & Permeable & Low bioavailability \\
\hline Myricetin & Natural product; flavonoid & Hsp70 inhibitor & Low toxicity & Permeable & \\
\hline Quercetin & Natural product; flavonoid & Hsp70 inhibitor & Low toxicity & Permeable & \\
\hline $\mathrm{B} 1 \mathrm{C} 11$ & N-Phenylamine & $\beta$-sheet inhibitor & Low toxicity & Unknown & \\
\hline B4A1 & N-Phenylamine & $\beta$-sheet inhibitor & Low toxicity & Unknown & \\
\hline B4D3 & N-Phenylamine & $\beta$-sheet inhibitor & Low toxicity & Unknown & \\
\hline B4D5 & N-Phenylamine & $\beta$-sheet inhibitor & Low toxicity & Unknown & \\
\hline Methylene blue & Phenothiazine & Hsp70 inhibitor & Low toxicity & Permeable & Blue color \\
\hline Quinoxalines & Phenothiazine & Hsp70 inhibitor & Selective toxicity & Permeable & Low bioavailability \\
\hline BSc3094 & Phenylthiazolyl-hydrazides & $\beta$-sheet inhibitor & Some toxicity & Unknown & \\
\hline Epalrestat & Rhodanine & $\beta$-sheet inhibitor & Some toxicity & Permeable & Aldose reductase inhibitor \\
\hline Troglitazone & Rhodanine & $\beta$-sheet inhibitor & Highly toxic & Likely & \\
\hline MKT-077 & Rhodocyanine & Hsp70 inhibitor & Selective toxicity & Not permeable & \\
\hline YM-01 & Rhodocyanine & Hsp70 inhibitor & Selective toxicity & Not permeable & \\
\hline YM-08 & Rhodocyanine & Hsp70 inhibitor & Selective toxicity & Permeable & Less potent than YM-01 \\
\hline Macrocycles & Synthetic & $\beta$-sheet inhibitor & Unknown & Unknown & \\
\hline
\end{tabular}

17-AAG, 17-(allylamino)-17-demethoxygeldamycin; AQ2S, anthraquinone-2-sulfonic acid; BBB, blood-brain barrier; C11, 3,3'-diethyl-9-methyl-thiacarbocyanine iodide; Cdc37, cell division control $37 \mathrm{kDa}$; Hsp, heat shock protein; MKT-077, 1-ethyl-2-((3-ethyl-5-(3-methylbenzothiazolin-2-yliden))-4-oxothiazolidin-2ylidenemethyl) pyridinium chloride; N744, 3,3'-bis( $\beta$-hydroxyethyl)-9-ethyl-5,5'-dimethoxythiacarbocyanine iodide; PR619, 2,6-diamino-3,5-dithiocyanopyridine, thiocyanic acid C,C'-(2,6-diamino-3,5-pyridinediyl) ester; 2,6-diaminopyridine-3,5-bis(thiocyanate).

\section{Assays and rationale for tau aggregation inhibitors}

Tau aggregation has been defined using multiple techniques, but three primary assays are traditionally used. Two of these techniques, the thioflavin fluorescence stain and the Gallyas silver stain, are typically used to examine tau aggregates in tissue. These stains bind beta sheets, allowing for measurements of tau amyloidogenicity [5]. Tau filaments are also often measured using electron microscopy, both in tissue and in vitro [6]. Biochemically, tau aggregation is measured using sequential extractions with detergents, such as sarkosyl and sodium dodecyl sulfate [7]. Currently, there is no direct measurement that can be performed to assess tau aggregation in living organisms; however, brain imaging using single photon emission computed tomography scans is in development.

In general, efforts aimed at either preventing or reversing tau aggregation are much further advanced than those targeting chaperones. Tau contains a well-characterized, 
aggregation-prone peptide sequence in the third microtubule binding repeat domain of exon $10+$ tau. This hexapeptide motif, VQIVYK, located at amino acids 306 to 311 , has been shown to exhibit the highest propensity to aggregate [8]. Drugs that directly bind near this hexapeptide region have been the most effective at preventing tau aggregation [9]. Interestingly, however this hexapeptide domain is also the region that chaperones most readily recognize [10]. This further suggests that there is some interplay between tau aggregation and its regulation by chaperones.

\section{Heat shock proteins as therapeutic targets}

Hsps are a group of molecular chaperones that assist in protein folding, transport, and degradation. These chaperones have been extensively studied for their ability to regulate aberrant intracellular proteins. Typically, misfolded tau collects in the soma and accumulates in the somatodendritic compartment $[11,12]$. Intracellular factors that can readily access this compartment are therapeutic options for preventing tau aggregation or degrading aberrant tau species. Molecular chaperones are naturally able to maintain a gateway that could determine the fate of a non-native protein, such as aggregated tau; however, with aging, this system begins to slow and proteins unnaturally accumulate. This ageinduced blockade can be caused by many factors, including dysfunctional or decreased degradation mechanisms [13] or declining chaperone levels coupled with rising levels of misfolded proteins [14].

The Hsp70, small Hsp, and Hsp90 families are notably known to play roles in tau pathology $[15,16]$. Currently, therapeutics targeting Hsp90 have shown promise as tau reducing agents $[17,18]$; however, a common problem with Hsp90 inhibitors is that they stimulate the transcription factor heat shock factor protein 1 (HSF1). Upon stress, HSF1 activation leads to induced expression of a whole panel of other Hsps that can antagonize the effects of Hsp90 inhibition. Hsp70 inhibitors have also shown promise as anti-tau therapeutics [19], and they do not cause a heat shock induction. However, there are more Hsp70 variants in the cell, each with different functions, and these inhibitors are not selective for one over the other. This non-specific inhibition could lead to unwanted side effects. There is also a need to increase blood-brain barrier (BBB) permeability of these compounds in order to create drugs that can be delivered peripherally yet treat centrally, a process that is in development [20].

\section{Hsp90 inhibition decreases tau}

The Hsp90 chaperone is a highly conserved, ATPrequiring protein that functions as part of a large protein complex. Among others, this complex includes the co- chaperones cell division control $37 \mathrm{kDa}$ (Cdc37), FK506 binding protein $51 \mathrm{kDa}$ (FKBP51), and carboxyl terminus of Hsc70-interacting protein (CHIP), all of which contain tetratricopeptide repeat domains that allow Hsp90 binding and aid in directing Hsp90 function. Since the original discovery that Hsp90 inhibitors could facilitate tau clearance $[17,18]$, many Hsp90 inhibitors have been identified as potential tau therapeutics. These inhibitors include radicicol and its multiple derivatives, geldanamycin, the geldanamycin analog 17-(allylamino)-17-demethoxygeldamycin (17-AAG), and $N-(7-((2 R, 3 R, 4 S, 5 R)-3,4-d i h y d r o x y-5-m e t h o x y-6,6-$ dimethyl-tetrahydro-2H-pyran-2-yloxy)-8-methyl-2-oxo-2Hchromen-3-yl)acetamide (KU-32). (For a comprehensive review of Hsp90 inhibitors, see [21]).

Of these inhibitors, geldanamycin has been the most studied as a tau therapeutic. An Hsp90-inhibiting antibiotic used to cause alterations in tau, geldanamycin has been shown to decrease levels of insoluble tau aggregates by $80 \%$ while leaving total tau levels unchanged [22]. Recently, a group showed that geldanamycin was able to activate the proteasome to cause tau degradation [23], and another study showed in a primary neuron model that geldanamycin treatment led to decreased tau phosphorylation by downregulating aberrant kinase activity [24]. Geldanamycin inhibits the Hsp90 homodimer by binding to its two N-terminal nucleotide binding domains [25]. However, geldanamycin proved to be quite toxic [26], prompting the development and testing of the geldanamycin analog 17-AAG.

\section{Recent developments of Hsp90 inhibitors}

Compared with its parent molecule, 17-AAG showed lower toxicity and had more potent effects at decreasing tau phosphorylation in primary neurons [24]. 17-AAG was also able to decrease tau in an in vitro model of tauopathy [27]. Although the analog compound was unable to alter tau phosphorylation at serines 396 and 404 or rescue a motor deficit, Sinadinos and colleagues recently showed that treating Drosophila larvae expressing human 3R tau with 17-AAG dramatically decreased total tau levels [28].

In addition to 17-AAG, radicicol is another Hsp90 inhibitor that was discovered after geldanamycin. Radicicol is a natural product that inhibits Hsp90 while inducing Hsp40 and Hsp70. Again in a Drosophila model, radicicol has been shown to dose-dependently decrease the levels of tau [28]. Analogs of radicicol, originally made for use in oncogenic research, have yet to be evaluated for their effects on tau [29].

Owing to the potentially toxic effects of $\mathrm{N}$-terminal Hsp90 ATPase inhibitors, C-terminal ATPase inhibitors are now thought to be preferred. These C-terminal inhibitors are currently in development through new research on novobiocin inhibitors. Novobiocin is an 
antibiotic that binds to the two C-terminal ATPase sites of the Hsp90 homodimer. Analogues of novobiocin were developed by the Blagg group to test whether $\mathrm{C}$-terminal ATPase inhibition of Hsp90 would yield fewer toxic side effects. From these studies, the new lead compound KU32 showed the greatest potential for efficacy against diseases of the central nervous system because it could cross the $\mathrm{BBB}$, and caused an attenuated heat shock response compared with N-terminal inhibitors [30,31]. The effects of KU-32 on tau biology in vivo have not yet been evaluated, but it appears to be a promising drug candidate for tauopathies.

Because inhibition of Hsp90 in many cases activates HSF1, it is very difficult to elucidate the mechanism by which Hsp90 inhibition decreases tau levels or aggregates. Additionally, as Hsp90 is involved in many diseases and is ubiquitously expressed, striving for one specific result through global Hsp90 inhibition may lead to many off-target effects. More effort has been placed recently on developing drugs that target co-chaperones of Hsp90 for increased specificity. This development, however, is in its infancy. Withaferin A, one known inhibitor of the Hsp90 co-chaperone Cdc37, was shown to decrease aggregated tau in a mouse model, but it also led to the induction of Hsp27 and Hsp70 [28]. Other Hsp90 co-chaperones, such as FKBP51 [32] and CHIP [22], have been identified as being good targets for altering tau phosphorylation states and levels. However, compounds directed at these targets have yet to be developed.

\section{Targeting Hsp70 with small molecules}

Along with the Hsp90 family, Hsp70 has been extensively studied as a therapeutic target for modulating tau $[15,22,33]$. The Hsp70 family is a ubiquitously expressed group that can prevent protein aggregation through ATP hydrolyses [34]. This family includes more than 10 members. The most commonly studied are the constitutively expressed Hsp73 (heat shock cognate (Hsc)70), the stress induced Hsp72 (Hsp70), glucose-regulated protein (GRP)78 (BiP), which is expressed in the endoplasmic reticulum, and mitochondrial GRP75 (mortalin) [35]. These $\sim 70 \mathrm{kDa}$ proteins have three functional domains: an N-terminal ATPase domain, a substrate binding domain, and a $\mathrm{C}$-terminal domain that functions as a lid.

Although many of the Hsp90 inhibitors discussed above can be also included in the Hsp70 modulating category because they induce Hsp70 expression, recent work from our group and others have identified Hsp70specific modulating drugs that can potently facilitate tau clearance $[20,33,36]$. Both activators and inhibitors of Hsp70 ATPase function have been identified, and these compounds appear to regulate the association of Hsp70 proteins with either DnaJ/Hsp40 proteins or nucleotide exchange factors, which in turn alters the ATPase activity of Hsp70. Currently, these compounds are derived from three drug families; the flavonoids, the phenothiazine dyes, and the rhodocyanine dyes [37,38]. Importantly, these compounds have few known side effects. Altering ATPase function has been shown to affect tau biology, so these Hsp70 ATPase modulators may be relevant targets for tauopathies.

\section{Flavonoids as anti-tau therapeutics}

Natural products research has yielded many unique, active compounds. A class of these known as flavonoids have had broad implications in many diseases. While the flavonoid myricetin possesses anti-Hsp70 activity and reduces tau levels [33], other flavonoids that lack antiHsp70 activity can still reduce tau levels, including curcumin [39] and quercetin [40]. Curcumin has been shown to alter tau phosphorylation in primary neurons [41]. Moreover, this yellow compound has recently been implicated to have therapeutic value in mouse models of tauopathies [42]. Just last year, curcumin was shown to elevate Hsp90 and Hsc70 without affecting HSF1, while suppressing soluble tau but not insoluble tau [43]. Moreover, curcumin is BBB permeable and showed a significant behavioral deficit rescue. Curcumin has been involved with many clinical trials and is well tolerated, but its efficacy in the treatment or prevention of tauopathies has yet to be clearly demonstrated. Quercetin has shown protective effects against amyloid-beta pathology and may have a therapeutic role for tau aggregation.

\section{Phenothiazines: efficacy through pleiotropy}

Phenothiazines have been studied for their therapeutic potential for over a century. One phenothiazine, methylene blue (MB; phenothiazine methylthionium chloride), has been involved in multiple clinical trials testing its efficacy for many diseases ranging from psychiatric disorders to cancer [44]. In addition, MB was originally identified as a potent beta-pleated sheet inhibitor [45] and more recently as an Hsp70 inhibitor [33].

Regardless of the mechanism, MB has been shown to be protective against tau accumulation in several models. Specifically, our group showed that MB given ad libitum in drinking water or through osmotic pump into the brain decreased tau phosphorylation and rescued learning impairment in $\mathrm{rTg} 4510$ mice [46]. This has since been validated by several other groups $[37,47,48]$. Another group showed that $\mathrm{MB}$ treatment on a tau $C$. elegans model alleviated tau-induced neuronal toxicity [49]. A proprietary formulation of $\mathrm{MB}$ has successfully passed through phase 1 and phase 2 clinical trials according to reports from TauRx Therapeutics (Singapore, Republic of Singapore) [50]. This year, the drug will go 
into phase 3 trials, for which patients are currently being recruited. The study is scheduled to conclude in 2015 [51].

Although $\mathrm{MB}$ is progressing in the clinic and has low toxicity, there are some drawbacks of this drug. $\mathrm{MB}$ is blue in color and causes discoloration of the eyes and urine; occasionally, it can also induce nausea.

Another member of the phenothiazine family, quinoxalines have a structure similar to MB. These compounds have been shown to have potent activity in preventing and even reversing the aggregation of tau. However, quinoxalines, while able to permeate the $\mathrm{BBB}$, were found to have very low absorption in vivo, making them inadequate as possible therapeutics for tau aggregation [39].

\section{Rhodocyanines: a promising Hsp70 inhibitor scaffold}

Rhodocyanine dyes have been studied for over a century for their therapeutic potential. Recent work from our group shows that one example of these dyes, 1-ethyl-2-((3-ethyl5-(3-methylbenzothiazolin-2-yliden))-4-oxothiazolidin-2ylidenemethyl) pyridinium chloride (MKT-077), can inhibit Hsp70 ATPase activity and facilitate tau clearance $[36,52,53]$. Researchers have been working to alter the chemical structure of MKT-077 to increase its potency, $\mathrm{BBB}$ permeability and target specificity, and to reduce its off-target effects [20,54].

A new family of MKT-077 compounds has been created, including the recently published YM compounds [20]. Two of these analogs, YM-01 and YM-08, showed anti-tau activity. Compared with the mostly mitochondrial MKT-077, YM-01 is more concentrated in the cytosol - potentially allowing it to be more accessible to cytosolic Hsp70 [54], conferring improved anti-tau efficacy. YM-08 is a further refinement of YM-01 that has reduced potency but dramatically improved BBB permeability [20].

\section{Small heat shock proteins: no enzymatic activity, yet major functionality}

Small Hsps are ATP-independent molecular chaperones with a molecular mass under $43 \mathrm{kDa}$. The small Hsps have two primary domains, one of which is an alpha crystalline C-terminal domain. These small Hsps are known to self-dimerize and oligomerize [55]. Upon a stress event, they become phosphorylated and dissociate to act upon non-native proteins [56].

Our group and others have shown that Hsp27 has relevant therapeutic potential. In vitro, Hsp27 was able to prevent the aggregation of tau filaments, as measured by dynamic light scattering and atomic force microscopy. Hsp27 is also able to prevent tau accumulation and rescue long-term potentiation deficits [16,36].

Hsp22 and Hsp25 have not been extensively explored for their effects on tau aggregation. However, they have been shown to prevent other amyloid accumulation such as amyloid-beta [57] and alpha-synuclein [58] aggregation. While compounds that regulate the activity of these small Hsps are not yet available, it is possible that they may be useful as therapeutics in recombinant form or via gene delivery. This is a developing area that could hold great promise for tauopathies.

\section{Small molecule inhibitors of tau aggregation}

While chaperone modulation of tau is an emerging field, preventing tau aggregation directly with compounds that bind tau is much further developed. Rhodanine drugs, including epalrestat and troglitazone, have been extensively developed and characterized by the Mandelkow group for their ability to alter tau aggregation. These low-toxicity compounds are actually able to disaggregate insoluble tau fibrils, known as paired helical filament tau [39]. This group performed an extensive characterization of over 50 derivatives, several of which proved to be potent compounds that prevented tau aggregation in neuronal cell lines [38]. Further developments of highly membrane permeable analogs are necessary to test these drugs in vivo.

Anthraquinones are synthetic, organic compounds that can also potently prevent and reverse tau aggregation [59]. A few derivatives that have exhibited anti-amyloidogenic properties include emodin, daunorubicin, mitoxantrone, and pixantrone [60]. Recently, the nontoxic analog anthraquinone-2-sulfonic acid (AQ2S) was identified to have not only anti-aggregation properties but was also found to be neuroprotective $[61,62]$. The structurally similar N-phenylamine also possesses anti-amyloidogenic properties. Derivatives of this compound, B1C11, B4D3, B4A1, and B4D5, have been shown to not only significantly prevent tau polymerization, but also to disassemble tau fibrils with relatively low toxicity [60]. However, there are no data that display the efficacy of these drugs in vivo.

Another aromatic scaffold, phenylthiazolyl-hydrazide was identified from a drug screen as a tau aggregation inhibitor. In one screen, the phenylthiazolyl-hydrazide derivative BSc3094 was identified to most effectively prevent and reverse tau aggregation out of dozens of phenylthiazolyl-hydrazide analogs produced, many of which showed some anti-amyloid activity. This family has low toxicity and their activity was also shown to be cytoprotective in a neuronal cell model of tauopathy [63].

Natural products have not only been shown to effect tau through chaperone modulation, as described above, but also through direct interaction with tau. Oleuropein, hydroxytyrosol, and oleuropein aglycone were isolated from olive extraction and showed anti-tau aggregation efficacy, with oleuropein having the greatest potency [5]. Future in vivo studies are needed to look at efficacy in the brain. Data suggest that oleuropein cannot pass the 
$\mathrm{BBB}$, but the aglycone analog has remained untested. Treatment of hydroxytyrosol, which is BBB permeable, in an in vivo tau mouse model would be necessary to determine aggregation inhibitory activity in the brain. Important to note is that administration of some phenols extracted from foods have been shown to exhibit different activity than that predicted in vivo [64].

Members in the carbocyanine scaffold, a group of blue-green dyes, have been shown to block tau aggregation; however, the potency of these drugs is variable. The structural composition of the linker chains in bisthiacarbocyanine derivatives was recently shown to lead to changes in the efficacy of aggregation inhibition [65]. One small molecule inhibitor in this family, 3,3'bis( $\beta$-hydroxyethyl)-9-ethyl-5,5' -dimethoxythiacarbocyanine iodide (N744), has been shown to have biphasic effects on tau aggregation [66]. This is not a surprising result, since other dye-based inhibitors are effective at preventing tau polymerization at specific concentrations, and not dose dependently [67]. N744 has additionally been shown to disaggregate tau filaments in a recombinant system [66]. Other analogs in this family, such as 3,3'diethyl-9-methyl-thiacarbocyanine iodide (C11), exhibited inhibitory aggregate activity in ex vivo tissue slices from a mouse line expressing human tau [68].

A new small molecule, 2,6-diamino-3,5-dithiocyanopyridine, thiocyanic acid C,C'-(2,6-diamino-3,5-pyridinediyl) ester, 2,6diaminopyridine-3,5-bis(thiocyanate) (PR-619), is an inhibitor of ubiquitin isopeptidases that upregulate Hsp70. An in vitro system showed that PR-619 was able to stabilize the microtubule network [69]. This same study showed that treatment led to small tau aggregates surrounding the microtubule organizing center. Importantly, tau phosphorylation at both serines 396 and 404 and serines 262 and 356 (12E8) was decreased, which increased the ability of tau to bind the microtubules [69]. Translation of this drug to an in vivo rodent model would be important to understand its therapeutic potential.

\section{Conclusion}

Tau aggregation contributes to the pathogenesis of many neurodegenerative diseases. Finding therapeutics that can prevent or reverse this non-native accumulation is thus highly desirable. Although many compounds have been recently identified to have anti-aggregative effects on tau, the large majority of these small molecule inhibitors is not specific for tau aggregates, but rather targets all proteins that can form beta-sheet amyloids. This lack of specificity may alter pharmacodynamics between individuals, making the appropriate dose difficult to assess based on the total amyloid burden in the body. The macrocyclic drugs are the first to be synthetically designed to specifically bind tau [9]. Further development of these compounds may help identify a tau specific inhibitor that is active in vivo.

Currently many aggregation inhibitors would need to be used at high concentrations to be effective against the high levels of tau present in neurons [70]. Tau is typically found in axons, but is thought to be further concentrated into somatodendritic aggregates in disease [71]. Delivering aggregation inhibitors with assistance from nanoparticle encapsulation [72] could therefore boost their efficacy by increasing their concentration in the brain. Other strategies that preferentially and specifically target tau aggregates within neurons or areas within the neuron where tau aggregates are present could also increase the potential for success. Perhaps combining therapies that specifically target tau aggregates and then facilitate tau clearance would further overcome this potential problem associated with high focal concentrations of tau.

The combination of tau aggregation inhibitors with compounds that can facilitate tau clearance could be advantageous in a clinical setting, possibly producing true synergy. Molecular chaperones are a prime target for regulating tau turnover; many drugs have been identified that alter the expression or activities of chaperone proteins, and advances in the last decade have increased drug efficacy and BBB permeability. These developments have allowed us to advance our understanding of the role of tau accumulation in disease, but concerns about specificity and off-target effects have slowed the progress of these compounds to the clinic. In addition, drugs that prevent aggregation, disaggregation, degradation, or increased expression may not be effective in preventing or reversing tauopathy phenotypes. Constructing the next generation of small molecule drugs to selectively eliminate only abnormal tau may be essential, a strategy that may now be possible given our advanced understanding of tau triage biology.

Note: This article is part of the series on Tau-based therapeutic
strategies, edited by Leonard Petrucelli. Other articles in this
series can be found at http://alzres.com/series/tau_therapeutics

\section{Abbreviations}

17-AAG: 17-(allylamino)-17-demethoxygeldamycin; AQ2S: Anthraquinone-2sulfonic acid; BBB: Blood-brain barrier; C11: 3,3'-diethyl-9-methyl-

thiacarbocyanine iodide; Cdc37: Cell division control 37 kDa; CHIP: Carboxyl terminus of Hsc70-interacting protein; FKBP51: FK506 binding protein 51 kDa; GRP: Glucose-regulated protein; HSF1: Heat shock factor protein 1; Hsc: Heat shock cognate; Hsp: Heat shock protein; KU-32: N-(7-((2R,3R,4S,5R)-3,4dihydroxy-5-methoxy-6,6-dimethyl-tetrahydro-2H-pyran-2-yloxy)-8-methyl-2-oxo$2 \mathrm{H}$-chromen-3-yl)acetamide; MB: Methylene blue (phenothiazine methylthionium chloride); MKT-077: 1-ethyl-2-((3-ethyl-5-(3-methylbenzothiazolin-2-yliden))4-oxothiazolidin-2-ylidenemethyl) pyridinium chloride; N744: 3,3'-bis( $\beta$ hydroxyethyl)-9-ethyl-5,5'-dimethoxythiacarbocyanine iodide; PR619: 2,6diamino-3,5-dithiocyanopyridine, thiocyanic acid C, $C^{\prime}$-(2,6-diamino-3,5pyridinediyl) ester, 2,6-diaminopyridine-3,5-bis(thiocyanate). 


\section{Competing interests}

The authors declare that they have no competing interests.

\section{Acknowledgements}

This work was supported by NIH/NINDS R01 NS073899.

\section{Published: 16 Sep 2013}

\section{References}

1. Hardy J, Orr H: The genetics of neurodegenerative diseases. J Neurochem 2006, 97:1690-1699.

2. Abisambra JF, Blair LJ, Hill SE, Jones J, Kraft C, Rogers J, Koren J, Jinwal UK, Lawson LY, Johnson AG, Wilcock D, O'Leary JC, Jansen-West K, Muschol M, Golde TE, Weeber EJ, Banko J, Dickey CA: Phosphorylation dynamics regulate $\mathrm{Hsp} 27-$ mediated rescue of neuronal plasticity deficits in tau transgenic mice. J Neurosci 2010, 30:15374-15382.

3. Voss K, Combs B, Patterson KR, Binder LI, Gamblin TC: Hsp70 alters tau function and aggregation in an isoform specific manner. Biochemistry 2012, 51:888-898.

4. Thompson AD, Scaglione KM, Prensner J, Gillies AT, Chinnaiyan A, Paulson $H L$, Jinwal UK, Dickey CA, Gestwicki JE: Analysis of the tau-associated proteome reveals that exchange of $\mathrm{Hsp70}$ for $\mathrm{Hsp} 90$ is involved in tau degradation. ACS Chem Biol 2012, 7:1677-1686.

5. Daccache A, Lion C, Sibille N, Gerard M, Slomianny C, Lippens G, Cotelle P: Oleuropein and derivatives from olives as Tau aggregation inhibitors. Neurochem Int 2011, 58:700-707.

6. Chang E, Congdon EE, Honson NS, Duff KE, Kuret J: Structure-activity relationship of cyanine tau aggregation inhibitors. J Med Chem 2009, 52:3539-3547.

7. Khlistunova I, Biernat J, Wang Y, Pickhardt M, von Bergen M, Gazova Z, Mandelkow E, Mandelkow EM: Inducible expression of Tau repeat domain in cell models of tauopathy: aggregation is toxic to cells but can be reversed by inhibitor drugs. J Biol Chem 2006, 281:1205-1214.

8. Bulic B, Pickhardt M, Schmidt B, Mandelkow EM, Waldmann H, Mandelkow E: Development of tau aggregation inhibitors for Alzheimer's disease. Angew Chem 2009, 48:1740-1752.

9. Zheng J, Liu C, Sawaya MR, Vadla B, Khan S, Woods RJ, Eisenberg D, Goux WJ, Nowick JS: Macrocyclic beta-sheet peptides that inhibit the aggregation of a tau-protein-derived hexapeptide. J Am Chem Soc 2011, 133:3144-3157.

10. Jinwal UK, Akoury E, Abisambra JF, O'Leary JC, Thompson AD, Blair LJ, Jin Y, Bacon J, Nordhues BA, Cockman M, Zhang J, Li P, Zhang B, Borysov S, Uversky VN, Biernat J, Mandelkow E, Gestwicki JE, Zweckstetter M, Dickey CA: Imbalance of Hsp70 family variants fosters tau accumulation. FASEB J 2013, 27:1450-1459.

11. Wolozin BL, Pruchnicki A, Dickson DW, Davies P: A neuronal antigen in the brains of Alzheimer patients. Science 1986, 232:648-650

12. Wood JG, Mirra SS, Pollock NJ, Binder LI: Neurofibrillary tangles of Alzheimer disease share antigenic determinants with the axonal microtubule-associated protein tau (tau). Proc Natl Acad Sci U S A 1986, 83:4040-4043

13. Massey AC, Zhang C, Cuervo AM: Chaperone-mediated autophagy in aging and disease. Curr Top Dev Biol 2006, 73:205-235.

14. Dickey C, Kraft C, Jinwal U, Koren J, Johnson A, Anderson L, Lebson L, Lee $D$, Dickson D, de Silva R, Binder LI, Morgan D, Lewis J: Aging analysis reveals slowed tau turnover and enhanced stress response in a mouse model of tauopathy. Am J Pathol 2009, 174:228-238.

15. Dou F, Netzer WJ, Tanemura K, Li F, Hartl FU, Takashima A, Gouras GK, Greengard $\mathrm{P}, \mathrm{Xu} \mathrm{H}$ : Chaperones increase association of tau protein with microtubules. Proc Natl Acad Sci U S A 2003, 100:721-726.

16. Shimura H, Miura-Shimura $Y$, Kosik KS: Binding of tau to heat shock protein 27 leads to decreased concentration of hyperphosphorylated tau and enhanced cell survival. J Biol Chem 2004, 279:17957-17962.

17. Dickey CA, Kamal A, Lundgren K, Klosak N, Bailey RM, Dunmore J, Ash P, Shoraka S, Zlatkovic J, Eckman CB, Patterson C, Dickson DW, Nahman NS Jr Hutton M, Burrows F, Petrucelli L: The high-affinity HSP90-CHIP complex recognizes and selectively degrades phosphorylated tau client proteins. J Clin Invest 2007, 117:648-658.

18. Luo W, Dou F, Rodina A, Chip S, Kim J, Zhao Q, Moulick K, Aguirre J, Wu N, Greengard P, Chiosis G: Roles of heat-shock protein 90 in maintaining and facilitating the neurodegenerative phenotype in tauopathies. Proc Natl Acad Sci U S A 2007, 104:9511-9516.

19. Jinwal UK, Koren J, O'Leary JC, Jones JR, Abisambra JF, Dickey CA: Hsp70 ATPase modulators as therapeutics for Alzheimer's and other neurodegenerative diseases. Mol Cell Pharmacol 2010, 2:43-46.

20. Miyata $Y$, Li X, Lee H-F, Jinwal UK, Srinivasan SR, Seguin SP, Young ZT, Brodsky JL, Dickey CA, Sun D, Gestwicki JE: Synthesis and initial evaluation of YM-08, a blood-brain barrier permeable derivative of the heat shock protein 70 (Hsp70) inhibitor MKT-077, which reduces tau levels. ACS Chem Neurosci 2013, 4:930-939.

21. Zhao H, Michaelis ML, Blagg BS: Hsp90 modulation for the treatment of Alzheimer's disease. Adv Pharmacol 2012, 64:1-25.

22. Petrucelli L, Dickson D, Kehoe K, Taylor J, Snyder H, Grover A, De Lucia M, McGowan E, Lewis J, Prihar G, Kim J, Dillmann WH, Browne SE, Hall A, Voellmy R, Tsuboi Y, Dawson TM, Wolozin B, Hardy J, Hutton M: CHIP and $\mathrm{Hsp70}$ regulate tau ubiquitination, degradation and aggregation. Hum Mol Genet 2004, 13:703-714

23. Opattova A, Filipcik P, Cente M, Novak M: Intracellular degradation of misfolded tau protein induced by geldanamycin is associated with activation of proteasome. J Alzheimers Dis 2013, 33:339-348.

24. Dou F, Yuan LD, Zhu JJ: Heat shock protein 90 indirectly regulates ERK activity by affecting Raf protein metabolism. Acta Biochim Biophys Sin 2005, 37:501-505.

25. Grenert JP, Sullivan WP, Fadden P, Haystead TA, Clark J, Mimnaugh E, Krutzsch H, Ochel HJ, Schulte TW, Sausville E, Neckers LM, Toft DO: The amino-terminal domain of heat shock protein 90 (hsp90) that binds geldanamycin is an ATP/ADP switch domain that regulates hsp90 conformation. J Biol Chem 1997, 272:23843-23850.

26. Ansar S, Burlison JA, Hadden MK, Yu XM, Desino KE, Bean J, Neckers L, Audus $\mathrm{KL}$, Michaelis ML, Blagg BS: A non-toxic Hsp90 inhibitor protects neurons from Abeta-induced toxicity. Bioorg Med Chem Lett 2007, 17:1984-1990.

27. Dickey CA, Koren J, Zhang YJ, Xu YF, Jinwal UK, Birnbaum MJ, Monks B, Sun M, Cheng JQ, Patterson C, Bailey RM, Dunmore J, Soresh S, Leon C, Morgan D, Petrucelli L: Akt and CHIP coregulate tau degradation through coordinated interactions. Proc Natl Acad Sci U S A 2008, 105:3622-3627.

28. Sinadinos C, Quraishe S, Sealey M, Samson PB, Mudher A, Wyttenbach A: Low endogenous and chemical induced heat shock protein induction in a 0N3Rtau-expressing Drosophila larval model of Alzheimer's disease. J Alzheimers Dis 2013, 33:1117-1133.

29. Soga S, Shiotsu Y, Akinaga S, Sharma SV: Development of radicicol analogues. Curr Cancer Drug Targets 2003, 3:359-369.

30. Li C, Ma J, Zhao H, Blagg BS, Dobrowsky RT: Induction of heat shock protein $70(\mathrm{Hsp} 70)$ prevents neuregulin-induced demyelination by enhancing the proteasomal clearance of c-Jun. ASN Neuro 2012, 4:e00102.

31. Lu Y, Ansar S, Michaelis ML, Blagg BS: Neuroprotective activity and evaluation of Hsp90 inhibitors in an immortalized neuronal cell line. Bioorg Med Chem 2009, 17:1709-1715.

32. Jinwal UK, Koren J 3rd, Borysov SI, Schmid AB, Abisambra JF, Blair $L$, Johnson AG, Jones JR, Shults CL, O'Leary JC 3rd, Jin Y, Buchner J, Cox MB, Dickey CA: The Hsp90 cochaperone, FKBP51, increases Tau stability and polymerizes microtubules. J Neurosci 2010, 30:591-599.

33. Jinwal UK, Miyata Y, Koren J 3rd, Jones JR, Trotter JH, Chang L, O'Leary J, Morgan D, Lee DC, Shults CL, Rousaki A, Weeber EJ, Zuiderweg ER, Gestwicki JE, Dickey CA: Chemical manipulation of hsp70 ATPase activity regulates tau stability. J Neurosci 2009, 29:12079-12088.

34. Wisen S, Gestwicki JE: Identification of small molecules that modify the protein folding activity of heat shock protein 70. Anal Biochem 2008 374:371-377.

35. Zuiderweg ER, Bertelsen EB, Rousaki A, Mayer MP, Gestwicki JE, Ahmad A: Allostery in the Hsp70 chaperone proteins. Top Curr Chem 2013, 328:99-153.

36. Abisambra J, Jinwal UK, Miyata Y, Rogers J, Blair L, Li X, Seguin SP, Wang L, Jin Y, Bacon J, Brady S, Cockman M, Guidi C, Zhang J, Koren J, Young ZT, Atkins CA, Zhang B, Lawson LY, Weeber EJ, Brodsky JL, Gestwicki JE, Dickey CA: Allosteric heat shock protein 70 inhibitors rapidly rescue synaptic plasticity deficits by reducing aberrant tau. Biol Psychiatry 2013, 74:367-374.

37. Akoury E, Pickhardt M, Gajda M, Biernat J, Mandelkow E, Zweckstetter M: Mechanistic basis of phenothiazine-driven inhibition of tau aggregation. Angew Chem 2013, 52:3511-3515.

38. Bulic B, Pickhardt M, Khlistunova I, Biernat J, Mandelkow EM, Mandelkow E, Waldmann H: Rhodanine-based tau aggregation inhibitors in cell models of tauopathy. Angew Chem 2007, 46:9215-9219. 
39. Bulic B, Pickhardt M, Mandelkow EM, Mandelkow E: Tau protein and tau aggregation inhibitors. Neuropharmacology 2010, 59:276-289.

40. Lu J, Wu DM, Zheng YL, Hu B, Zhang ZF, Shan Q, Zheng ZH, Liu CM, Wang YJ: Quercetin activates AMP-activated protein kinase by reducing PP2C expression protecting old mouse brain against high cholesterol-induced neurotoxicity. J Pathol 2010, 222:199-212.

41. Narlawar R, Pickhardt M, Leuchtenberger S, Baumann K, Krause S, Dyrks T, Weggen S, Mandelkow E, Schmidt B: Curcumin-derived pyrazoles and isoxazoles: Swiss army knives or blunt tools for Alzheimer's disease? ChemMedChem 2008, 3:165-172.

42. Ma QL, Yang F, Rosario ER, Ubeda OJ, Beech W, Gant DJ, Chen PP, Hudspeth B, Chen C, Zhao Y, Vinters HV, Frautschy SA, Cole GM: Betaamyloid oligomers induce phosphorylation of tau and inactivation of insulin receptor substrate via c-Jun $\mathrm{N}$-terminal kinase signaling: suppression by omega-3 fatty acids and curcumin. J Neurosci 2009, 29:9078-9089

43. Ma QL, Zuo X, Yang F, Ubeda OJ, Gant DJ, Alaverdyan M, Teng E, Hu S, Chen PP, Maiti P, Teter B, Cole GM, Frautschy SA: Curcumin suppresses soluble tau dimers and corrects molecular chaperone, synaptic, and behavioral deficits in aged human tau transgenic mice. J Biol Chem 2013, 288:4056-4065.

44. Schirmer RH, Adler H, Pickhardt M, Mandelkow E: 'Lest we forget you methylene blue...'. Neurobiol Aging 2011, 32(2325):e7-e16.

45. Wischik CM, Edwards PC, Lai RY, Roth M, Harrington CR: Selective inhibition of Alzheimer disease-like tau aggregation by phenothiazines. Proc Natl Acad Sci U S A 1996, 93:11213-11218.

46. O'Leary JC 3rd, Li Q, Marinec P, Blair LJ, Congdon EE, Johnson AG, Jinwal UK, Koren J 3rd, Jones JR, Kraft C, Peters M, Abisambra JF, Duff KE, Weeber EJ, Gestwicki JE, Dickey CA: Phenothiazine-mediated rescue of cognition in tau transgenic mice requires neuroprotection and reduced soluble tau burden. Mol Neurodegener 2010, 5:45.

47. Congdon EE, Wu JW, Myeku N, Figueroa YH, Herman M, Marinec PS, Gestwicki JE, Dickey CA, Yu WH, Duff KE: Methylthioninium chloride (methylene blue) induces autophagy and attenuates tauopathy in vitro and in vivo. Autophagy 2012, 8:609-622.

48. Brunden KR, Trojanowski JQ, Lee VM: Advances in tau-focused drug discovery for Alzheimer's disease and related tauopathies. Nat Rev Drug Discov 2009, 8:783-793.

49. Fatouros C, Pir GJ, Biernat J, Koushika SP, Mandelkow E, Mandelkow EM, Schmidt E, Baumeister R: Inhibition of tau aggregation in a novel Caenorhabditis elegans model of tauopathy mitigates proteotoxicity. Hum Mol Genet 2012, 21:3587-3603.

50. Wischik C, Staff R: Challenges in the conduct of disease-modifying trials in AD: practical experience from a phase 2 trial of Tau-aggregation inhibitor therapy. J Nutr Health Aging 2009, 13:367-369.

51. Safety and efficacy study evaluating TRX0237 in subjects with mild to moderate alzheimer's disease. [http://www.clinicaltrials.gov/ct2/show/NCT01689246? term=alzheimer\%27s\&rank=10]

52. Miyata Y, Koren J, Kiray J, Dickey CA, Gestwicki JE: Molecular chaperones and regulation of tau quality control: strategies for drug discovery in tauopathies. Future Med Chem 2011, 3:1523-1537.

53. Rousaki A, Miyata Y, Jinwal UK, Dickey CA, Gestwicki JE, Zuiderweg ER: Allosteric drugs: the interaction of antitumor compound MKT-077 with human Hsp70 chaperones. J Mol Biol 2011, 411:614-632.

54. Koren J 3rd, Miyata Y, Kiray J, O'Leary JC 3rd, Nguyen L, Guo J, Blair LJ, Li X, Jinwal UK, Cheng JQ, Gestwicki JE, Dickey CA: Rhodacyanine derivative selectively targets cancer cells and overcomes tamoxifen resistance. PLOS One 2012, 7:e35566.

55. Lelj-Garolla B, Mauk AG: Self-association of a small heat shock protein. J Mol Biol 2005, 345:631-642.

56. Koteiche HA, McHaourab HS: Mechanism of chaperone function in small heat-shock proteins. Phosphorylation-induced activation of two-mode binding in alphaB-crystallin. J Biol Chem 2003, 278:10361-10367.

57. Wilhelmus MM, de Waal RM, Verbeek MM: Heat shock proteins and amateur chaperones in amyloid-beta accumulation and clearance in Alzheimer's disease. Mol Neurobiol 2007, 35:203-216.

58. Bruinsma IB, Bruggink KA, Kinast K, Versleijen AA, Segers-Nolten IM, Subramaniam V, Bea Kuiperij H, Boelens W, de Waal RM, Verbeek MM: Inhibition of alpha-synuclein aggregation by small heat shock proteins. Proteins 2011, 79:2956-2967.
59. Jackson TC, Verrier JD, Kochanek PM: Anthraquinone-2-sulfonic acid (AQ2S) is a novel neurotherapeutic agent. Cell Death Dis 2013, 4:e451.

60. Pickhardt M, Biernat J, Khlistunova I, Wang YP, Gazova Z, Mandelkow EM, Mandelkow E: N-phenylamine derivatives as aggregation inhibitors in cell models of tauopathy. Curr Alzheimer Res 2007, 4:397-402.

61. Pickhardt M, Gazova Z, von Bergen M, Khlistunova I, Wang Y, Hascher A Mandelkow EM, Biernat J, Mandelkow E: Anthraquinones inhibit tau aggregation and dissolve Alzheimer's paired helical filaments in vitro and in cells. J Biol Chem 2005, 280:3628-3635.

62. Convertino M, Pellarin R, Catto M, Carotti A, Caflisch A: 9,10-Anthraquinone hinders beta-aggregation: how does a small molecule interfere with Abeta-peptide amyloid fibrillation? Protein Sci 2009, 18:792-800.

63. Pickhardt M, Larbig G, Khlistunova I, Coksezen A, Meyer B, Mandelkow EM, Schmidt B, Mandelkow E: Phenylthiazolyl-hydrazide and its derivatives are potent inhibitors of tau aggregation and toxicity in vitro and in cells. Biochemistry 2007, 46:10016-10023.

64. Acin S, Navarro MA, Arbones-Mainar JM, Guillen N, Sarria AJ, Carnicer R, Surra JC, Orman I, Segovia JC, Torre Rde L, Covas MI, Fernández-Bolaños J, Ruiz-Gutiérrez V , Osada J: Hydroxytyrosol administration enhances atherosclerotic lesion development in apo E deficient mice. J Biochem 2006, 140:383-391.

65. Schafer KN, Murale DP, Kim K, Cisek K, Kuret J, Churchill DG: Structureactivity relationship of cyclic thiacarbocyanine tau aggregation inhibitors. Bioorg Med Chem Lett 2011, 21:3273-3276.

66. Necula M, Chirita CN, Kuret J: Cyanine dye N744 inhibits tau fibrillization by blocking filament extension: implications for the treatment of tauopathic neurodegenerative diseases. Biochemistry 2005, 44:10227-10237.

67. Congdon EE, Necula M, Blackstone RD, Kuret J: Potency of a tau fibrillization inhibitor is influenced by its aggregation state. Arch Biochem Biophys 2007, 465:127-135

68. Congdon EE, Figueroa YH, Wang L, Toneva G, Chang E, Kuret J, Conrad C, Duff KE: Inhibition of tau polymerization with a cyanine dye in two distinct model systems. J Biol Chem 2009, 284:20830-20839.

69. Seiberlich V, Goldbaum O, Zhukareva V, Richter-Landsberg C: The small molecule inhibitor PR-619 of deubiquitinating enzymes affects the microtubule network and causes protein aggregate formation in neural cells: implications for neurodegenerative diseases. Biochim Biophys Acta 1823, 2012:2057-2068

70. Binder LI, Frankfurter A, Rebhun LI: The distribution of tau in the mammalian central nervous system. J Cell Biol 1985, 101:1371-1378.

71. Wang YP, Biernat J, Pickhardt M, Mandelkow E, Mandelkow EM: Stepwise proteolysis liberates tau fragments that nucleate the Alzheimer-like aggregation of full-length tau in a neuronal cell model. Proc Natl Acad Sci U S A 2007, 104:10252-10257.

72. Grabrucker AM, Garner CC, Boeckers TM, Bondioli L, Ruozi B, Forni F, Vandelli MA, Tosi G: Development of novel $\mathrm{Zn}^{2+}$ loaded nanoparticles designed for cell-type targeted drug release in CNS neurons: in vitro evidences. PLoS One 2011, 6:e17851.

\subsection{6/alzrt207}

Cite this article as: Blair et al:: Potential synergy between tau aggregation inhibitors and tau chaperone modulators. Alzheimer's Research \& Therapy 2013, 5:41 